\title{
Myelography in the Assessment of Degenerative Lumbar Scoliosis and Its Influence on Surgical Management
}

George McKay, Peter Alexander Torrie, Wendy Bertram, Priyan Landham, Stephen Morris, John Hutchinson, Roland Watura, lan Harding

Department of Spinal Surgery, Southmead Hospital, Bristol, United Kingdom

\section{Corresponding Author:}

George McKay

Department of Spinal Surgery, Southmead Hospital, Southmead Road, Westbury-on-Trym, Bristol, BS10 5NB, United Kingdom

Tel: +44-117-950-5050

E-mail: mr.g.mckay@googlemail.com

Received: August 2, 2017

Revised: October 25, 2017

Accepted: November 6, 2017

Objective: Myelography has been shown to highlight foraminal and lateral recess stenosis more readily than computed tomography $(\mathrm{CT})$ or magnetic resonance imaging (MRI). It also has the advantage of providing dynamic assessment of stenosis in the loaded spine. The advent of weight-bearing MRI may go some way towards improving assessment of the loaded spine and is less invasive, however availability remains limited. This study evaluates the potential role of myelography and its impact upon surgical decision making.

Methods: Of 270 patients undergoing myelography during 2006-2009, a period representing peak utilisation of this imaging modality in our unit, we identified 21 patients with degenerative scoliosis who fulfilled our inclusion criteria. An operative plan was formulated by our senior author based initially on interpretation of an MRI scan. Subsequent myelogram and CT myelogram investigations were scrutinised, with any additional abnormalities noted and whether these impacted upon the operative plan.

Results: From our 21 patients, $18(85.7 \%)$ had myelographic findings not identified on MRI. Of note, in 4 patients, supine CT myelography yielded additional information when compared to supine MRI in the same patients. The management of 7 patients (33\%) changed as a result of myelographic investigation. There were no complications of myelography of the total 270 analysed.

Conclusion: MRI scan alone understates the degree of central and lateral recess stenosis. In addition to the additional stenosis displayed by dynamic myelography in the loaded spine, we have also shown that static myelography and CT myelography are also invaluable tools with regards to surgical planning in these patients.

Key Words: Degenerative, Deformity, Myelography, Imaging, Magnetic resonance imaging

\section{INTRODUCTION}

The prevalence of degenerative lumbar scoliosis (DLS) has been reported to range between $3.14 \%-68 \% \%^{8,13,24,28,30)}$, and is known to increase with advancing age ${ }^{13,14,18,20)}$. The clinical presentation and demands of this patient cohort is broad, heterogeneous and often and associated with complex local deformities (antero, retro, lateral, and rotational listheses). Potentially treatable, these can be mobile or fixed, and may cause dynamic or static stenosis. This makes decision-making and operative planning a complex challenge. Spinal surgeons must consider the merits of limited versus extensive decompression, whether to perform short or long fusion, and what combinations of these are appropriate in each individual patient.

In the era before 3-dimensional imaging, myelography was historically described as "an essential preoperative measure" for patient as- sessment and preoperative planning ${ }^{4)}$. More recently improved availability of computed tomography (CT) and magnetic resonance imaging (MRI) has resulted in a reduction in the use of myelography as a diagnostic tool. These imaging modalities provide cross sectional information and are often considered relatively 'low risk' investigations by most clinicians. The available literature presents mixed conclusions on the value of myelography compared with these other imaging modalities and will be discussed in this work.

We sought in particular to assess what, if any, additional information that was conveyed by dynamic myelography and subsequent supine CT myelography when compared to findings obtained on supine MRI. Additional data was collected regarding complications of myelography, and how any additional findings of myelography changed management. As a result of this we propose criteria for requesting myelography in the setting of degenerative scoliosis. 


\section{MATERIALS AND METHODS}

We retrospectively reviewed the MRI lumbar spine and myelography imaging of all patients in our practice between 2006 and 2009, a period representing peak utilisation of this imaging modality in our unit. Following identification of our patient COhort the medical notes of each suitable patient were reviewed, including electronic outpatient clinic letters and Multidisciplinary Team (MDT) meeting documentation. The study inclusion criteria were; patients older than 45 years of age, presence of a DLS, patients with MRI lumbar spine and myelography investigations performed within 12 months of one another, and patients who following this received operative intervention. In addition, clear documentation regarding investigation findings and the decision making process following MRI scan and then subsequent to myelographic intervention was essential, and this was obtained by thorough review of patient notes or electronic clinic letters. A surgical plan must have been clear at both of these time points. This limited the number of patients included in the study significantly, but this was offset by our ability to ensure that there was a clear change to patient management due to the addition of myelographic investigation.

The presence of a DLS was determined by evidence of coronal plain deformity of greater than $10^{\circ}$ between the superior endplate of L1 to the superior endplate of S1 on the standing antero-posterior (AP) plain film lumbar spine radiograph.

For all of the patients in our cohort the indication for myelography was the presence of neurological symptoms not explained by other available imaging modalities in the setting of DLS. All patients were being considered as potential operative candidates during the period of investigation and all received operative intervention when investigations were complete. Since the indications for both myelographic investigation and MRI scanning predated our study, we did not require approval from our Institutional Review Board or Ethics Committee. Other indications for myelography in our unit include patients with metalwork in whom neural structures are not well seen due to artefact and rarely, the unexplained radicular pain in the presence of a normal supine static MRI and standing X-ray.

In terms of the surgical decision making process, each case was presented in the forum of our MDT meeting and as such the imaging modalities were reviewed and discussed at length with the benefit of interpretation by at least the lead author $(\mathrm{IJH})$ and another author (RW) who are consultants in spinal surgery and radiology respectively. Several additional consultant clinicians from both specialties are present during such meetings in addition to this. Each imaging modality therefore had a consensus agreement on findings so as to minimise interpretation errors.

Prior to reviewing the results of myelography the senior author had determined a preliminary operative plan, based on the patient's symptoms, examination, standing X-rays and MRI scans which were also discussed in a previous MDT. When reviewing the myelography imaging, we noted positive findings from each investigation, recording also the position of the patient (supine, prone, weight bearing, and stress) in which the radiological findings where observed.

The results of MRI scanning were then compared with the results of myelography in the MDT. Significant differences were noted, for example; absence of stenosis or spondylolisthesis on MRI investigation but objective evidence on myelography. All patients undergoing dynamic myelography subsequently underwent supine CT myelography as part of our units' myelography protocol. Significant anatomical findings were also collated from this CT and compared with MRI lumbar spine investigations in a similar fashion to the plain film myelography.

Any change in this operative plan following myelography was noted. If patients with intrusive symptoms were being investigated by myelography in search of a surgical target due to a previously entirely "normal" MRI scan, this was also noted.

Each myelogram was performed or supervised by the consultant musculoskeletal radiologists within our department. Patients were fully consented, the procedure was explained to the patient including the commonest complications and side effects (including; headaches, cerebrospinal fluid leak, infection, bleeding, amongst others). Written consent was obtained. A detailed Nursing Procedure Care Plan was carried out and the details were recorded.

The procedure was carried out using fluoroscopic guidance with the patient positioned prone. Full aseptic technique was used. Local anaesthetic in the form of plain Lidocaine (1\%) was infiltrated to anaesthetise the skin. A 22-G spinal needle was advanced between the spinous process at a level below the conus, depending on review of previous imaging. Lateral view fluoroscopic screening was performed to assess needle depth. Contrast was injected to confirm needle position (Omnipaque 240). A total of $8-\mathrm{mL}$ contrast was then injected.

Screening views were taken supine, supine right and left oblique, plus lateral. Then varying degrees rising for example 30 and 45 degrees - supine, supine right and left oblique. This was followed by erect supine, supine right and left oblique, then bending right and left lateral, and finally sagittal view erect plus forward flexion and extension.

Patients then proceeded to CT - (images plus reconstructions) axial, coronal, sagittal. Oblique reconstructions were taken if necessary. Scans were performed on a 0.6 collimator. Data was reconstructed in 3 planes using 2-mm width slices at 2-mm intervals on a bony window.

\section{RESULTS}

Two hundred seventy myelograms were performed during the study period. Indications for myelogram were claustrophobia, metalwork in situ prohibiting MRI, symptoms not explained by MRI/X-Ray/Anaesthetic Blocks, amongst others. We noted during this period there were zero complications of myelography in our unit using the described technique and modern contrast media.

Twenty-one patients (17 female patients) fulfilled the inclusion 
criteria. Mean (range) age is $68(45-82)$ years. The mean interval between investigations was 5.5 months, range $0-12$. Eighteen myelograms (86\%) revealed findings not seen on MRI. Fifteen patients had a single new abnormality, 1 patient had 2 abnormalities, and in 2 patients there were 3 new abnormalities. Additional abnormalities observed on myelography and not on the lumbar MRI investigation were inclusive of; facet/ligamentous bulging in 13 patients, 1 spondylolisthesis (Fig. 1), 1 retrolisthesis (on extension), and 1 lateral subluxation (Fig. 2). In 4 cases the facet/ligamentous bulging was more pronounced on myelography compared with MRI. This was notably also evident on the supine myelogram views. We found that stress views in the coronal plane during myelography conferred no additional information in this study. We also noted that the listheses were also seen on weight bearing plain radiographs but not on MRI.

On subsequent CT myelography, 2 cases showed new findings not seen on MRI or plain myelography. These were a foraminal osteophyte and a pars defect. In total, the management of $7 \mathrm{pa}-$ tients changed as a result of myelography/CT myelography. A summary of these findings and how surgical management changed as a result is presented in Table 1. On reviewing follow-up clinic correspondence for this cohort of 7 patients, each patient experienced a subjective and objective improvement in preoperative symptoms following the operative interventions described. At the time of data collection no patient in this group had received any further operative intervention.

We had no cases of infection during our study period, however since the study was completed there has been one episode of meningitis in our unit, requiring treatment with intravenous antibiotics, with a full subsequent recovery. We have therefore found the complication profile to be low, but not zero, and this warrants consideration when requesting this investigation.

\section{DISCUSSION}

Degenerative scoliosis is a common, treatable condition and is associated with stenosis. We accept that in some instances the accepted standard for evaluation of stenosis is currently MRI, however this is theoretically limited due to its supine, static nature. MRI may display high quality imaging of the supine patient, however it does not provide information on what is happening to the spinal anatomy when the patient experiences pain,
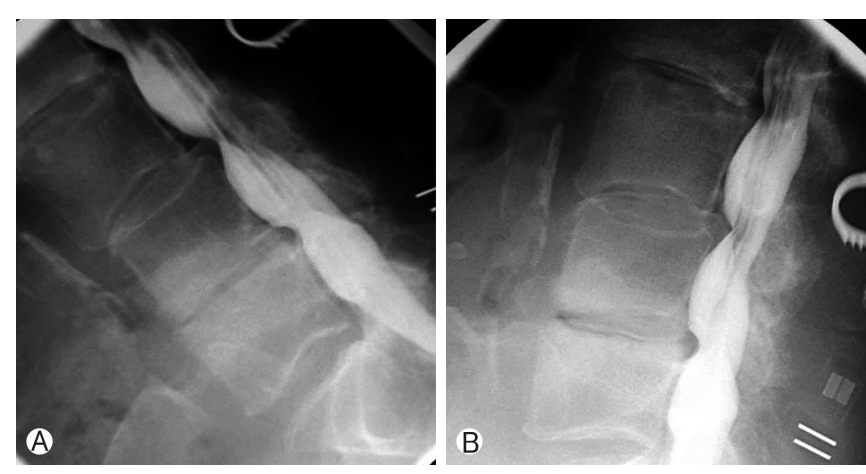

Fig. 1. Flexion-slip and compression (A) and extension-reduction with reduced stenosis (B) myelography displaying dynamic compression.

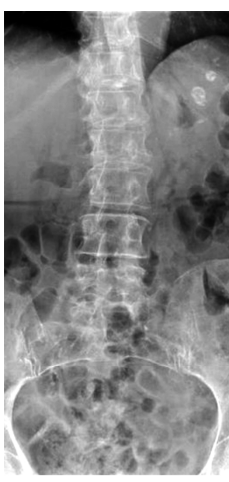

Prone

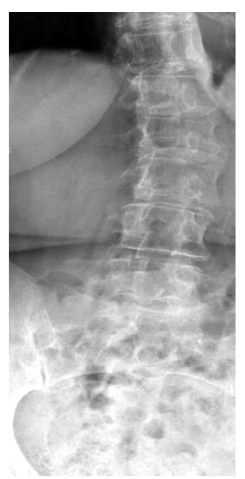

Erect

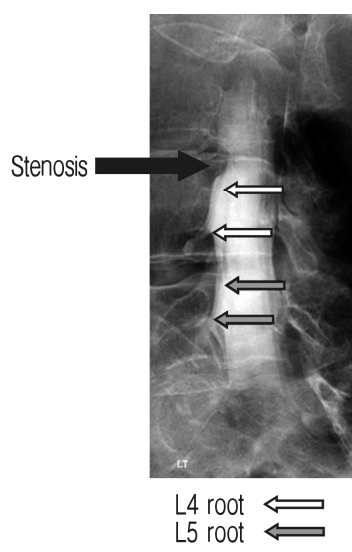

Fig. 2. Example of lateral subluxation - visible on weight bearing $\mathrm{X}$ ray but not on supine X-ray (or magnetic resonance imaging). The stenosis is clearly visible on the myelogram. The patient was complaining of right L4 radicular pain.

Table 1. Summary of myelogram/CT myelogram findings that were not present on MRI and how surgical management was affected by the results

\begin{tabular}{ll}
\hline \hline $\begin{array}{l}\text { Findings on myelography NOT present on MRI } \\
\text { (No. of cases) }\end{array}$ & \multicolumn{1}{c}{ Implication on surgical management } \\
\hline Dynamic stenosis at adjacent proximal level (2) & $\begin{array}{l}\text { Proximal extension of fusion without entering canal. } \\
\text { Dynamic stenosis distally (1) }\end{array}$ \\
Static stenosis not seen on MRI (2) & $\begin{array}{l}\text { One Isolated decompression. Alternatively patient would not have been offered surgery. } \\
\text { One decompression of canal with long fusion. Alternatively canal would not have been } \\
\text { entered. }\end{array}$ \\
Lateral subluxation with stenosis (1) & $\begin{array}{l}\text { Instrumentation and decompression performed. Alternatively patient would have received } \\
\text { decompression only. }\end{array}$ \\
2-Level dynamic stenosis not seen on MRI (1) & $\begin{array}{l}\text { 2-Level fusion performed without entering canal. Alternatively patient would not have been } \\
\text { offered surgery. }\end{array}$ \\
\hline
\end{tabular}

CT, computed tomography; MRI, magnetic resonance imaging. 
for example when standing. Myelography provides this option due to its dynamic nature.

Myelography allows for the dynamic assessment of the spine under physiological loading, including forward flexion, lateral flexion and extension. Furthermore the patient is able to relay their symptomatology with specific reference to positional exacerbation. Diagnostic specificity has been previously shown to increase when subjecting patients to axial loading during imaging $^{29)}$. We accept that CT or MRI remain the gold standard in some diagnostic scenarios, Ossified Posterior Longitudinal Ligament ${ }^{10)}$ and far lateral disc herniations ${ }^{9)}$ being good examples of such instances. Myelography has however been shown to offer more information than MRI in presurgical diagnosis of symptomatic foraminal stenosis ${ }^{1)}$ and highlights lateral recess stenosis more readily than MRI or CT alone. Epstein found that these findings changed the extent of decompression performed during surgery in $72 \%$ of patients ${ }^{5}$.

The recent evidence emerging regarding upright MRI scanning has contributed to the value of dynamic imaging, with studies showing a positive correlation between symptoms, dural sac cross sectional area, and the degree of lumbar spinal canal stenosis $^{12)}$. It has also been demonstrated that standing MRI offers superior imaging to recumbent MRI in the case of cervical and lumbosacral disc herniation, as well as in anterior spondylolisthesis ${ }^{6}$. . Recent work by Kanno et al. ${ }^{11)}$ examined the relationship between supine MRI, upright MRI and weight bearing myelography. They found upright MRI had higher sensitivity and specificity in lumbar spinal canal stenosis than supine MRI, and demonstrated that upright MRI can demonstrate similar positional changes of the dural sac size as demonstrated by weight bearing myelography.

In units such as our own where standing MRI scan remains unavailable, consideration should therefore be given to the efficacy of currently available technologies. The specific role of myelographic investigation as an adjunct to MRI has been explored in recent work from Germany ${ }^{16,25)}$ and Japan ${ }^{17,23)}$. This body of evidence appears to show that in degenerative disease of the Lumbar spine, weight bearing myelographic investigation yields additional diagnostic information compared with supine MRI, and is itself superseded by dynamic myelographic investigation such as myelography performed with extension. A review of the United Kingdom literature reveals only one case report article examining the role and utility of myelography as an adjunct to $\mathrm{MRI}^{3)}$, which appears to offer support to the above literature.

Our findings are in agreement with previous radiological studies demonstrating that space within the spinal canal is posture dependent ${ }^{19)}$, and if imaging is performed under loaded conditions, diagnostic specificity is increased ${ }^{29)}$. In $86 \%$ of out cohort, myelography enhanced our diagnostic ability. The majority of additional abnormalities detected comprised primarily of pathologies which are influenced by loading. It is worth noting however, that in 4 of our patients, supine myelography also proved to be superior to supine MRI. This suggests there are further merits with respect to myelography beyond the advantages offered by load bearing. Our study also suggests that MRI scanning alone underestimates the true extent of central and lateral recess stenosis. The use of CT myelography provided additional useful information regarding the true nature of stenosis, particularly the contribution of bony rather than soft tissue lesions. With the ability to arrange the cuts of CT to fit around the deformity, there are further advantages that become apparent in imaging complex deformities. It would appear that our United Kingdom based study supports the afore-mentioned overseas proponents of myelography as a useful adjunct to MRI, particularly in complex patients with unexplained symptoms using alternative imaging modalities $3,16,17,23,25)$.

We have also shown that myelography improved diagnostic accuracy, allowing us to more comprehensively address the patient's needs. If no culprit lesion is identified on imaging, patients may quite reasonably be denied the prospect of surgical intervention, their clinician outweighing the risks of surgery against the prospect of treating symptoms without hard diagnostic evidence following investigation. Patients with established abnormalities on MRI scanning may too benefit from concomitant myelographic investigation, with further information to be gained with regard to fusion or decompression levels. With an enhanced ability to avoid unnecessarily entering the canal for example, surgeons may be able to further minimise potential complications.

In the setting of degenerative scoliosis, our study highlights that myelography not only provides additional radiological information compared with MRI alone, but also that in some cases this can directly lead to a change in operative strategy. There is therefore a theoretical potential to improve clinical outcome but this needs to be offset against the potential low risk and resource of an invasive investigation.

Myelography is a more invasive procedure than MRI and CT and although not common, there is potential for complications. Postiacchini found a postmyelography complication rate of $37 \%$ for inpatients and $40 \%$ for outpatients ${ }^{21)}$. The majority of these complications were mild headache, and the incidence was reduced in patients who received a lower dose of contrast medium. In 2000, Suess et al. ${ }^{27}$ published a case report and a review of 16 previously reported cases documenting subacute intracranial subdural haematoma following myelography. Although rare, cases of post myelography seizure have been reported ${ }^{2,15,26}$. An email survey of the American Society of Neuroradiology found that 82\% of responding members (response rate 15\%) reported no significant complications in the past 5 years ${ }^{22}$. Notably, Herkowitz et al. $^{7)}$ found the incidence of postmyelography side effects was higher in women and that patients presenting with a subjective complaint along with a negative clinical examination and negative myelogram, reported a much higher rate of side effects such as nausea, vomiting, and headaches.

One limitation of our study is that we had no comparative control group, and as such we are unable to directly measure how this affected our patient outcomes. It is worth noting however that in our cohort that at minimum 2-year follow-up none of the patients in our cohort required revision operation for recurrent or residual pain. We recognise there are other limitations to consider with our study. Interpretation of myelography 
is subjective, and an accurate quantitative assessment is not possible. To mitigate against this we measured the presence and absence of abnormalities rather than the quantity of abnormality. The time lapse between investigations should be noted, since in order to exclude development of interval pathology both investigations would ideally be performed in succession. Although MRI scanning is still considered as the gold standard investigation, its main limitation is that it is currently commonly performed as a supine and static investigation. Upright MRI does have apparent advantages over supine MRI but has its own limitations due to availability, time taken, and difficulties obtaining the correct cuts to demonstrate levels/pathology adequately. It may also be poorly tolerated by a suffering patient who by definition finds it difficult to stand.

We suggest using myelography selectively in degenerative scoliosis when neurological symptoms are not explained by what is seen on standing X-rays or MRI scanning. In our practice, it is also an invaluable tool in patients with non-MRI-compatible implants or in whom MRI scanning is not possible.

\section{CONCLUSION}

We have shown that the extent of surgical treatment offered to patients is affected by using myelographic investigation in addition to MRI scanning preoperatively. In the current setting where standing MRI remains an emerging technology, we would encourage clinicians to consider the merits of myelography as a useful adjunct to diagnosis and surgical planning in the setting of DLS.

\section{CONFLICT OF INTEREST}

No potential conflict of interest relevant to this article was reported.

\section{REFERENCES}

1. Aota Y, Niwa T, Yoshikawa K, Fujiwara A, Asada T, Saito T: Magnetic resonance imaging and magnetic resonance myelography in the presurgical diagnosis of lumbar foraminal stenosis. Spine 32: 896-903, 2007

2. Dalen K, Kerr HH, Wang AM, Olson RE, Wesolowski DP, Farah J: Seizure activity after iohexol myelography. Spine 16:384, 1991

3. Eadsforth T, Niven S, Barrett C: The utility of myelography in lumbar canal stenosis. Br J Neurosurg 26:578-579, 2012

4. Epstein JA, Epstein BS, Jones MD: Symptomatic lumbar scoliosis with degenerative changes in the elderly. Spine 4:542-547, 1979

5. Epstein NE, Epstein JA, Carras R, Hyman RA: Far lateral lumbar disc herniations and associated structural abnormalities. An evaluation in 60 patients of the comparative value of CT, MRI, and myelo-CT in diagnosis and management. Spine 15:534-539, 1990

6. Ferreiro Perez A, Garcia Isidro M, Ayerbe E, Castedo J, Jinkins JR: Evaluation of intervertebral disc herniation and hypermobile intersegmental instability in symptomatic adult patients undergoing recumbent and upright MRI of the cervical or lumbosacral spines. Eur J Radiol 62:444-448, 2007
7. Herkowitz HN, Romeyn RL, Rothman RH: The indications for metrizamide myelography. Relationship with complications after myelography. J Bone Joint Surg Am 65:1144-1149, 1983

8. Hong JY, Suh SW, Modi HN, Hur CY, Song HR, Park JH: The prevalence and radiological findings in 1347 elderly patients with scoliosis. J Bone Joint Surg Br 92:980-983, 2010

9. Jackson RP, Glah JJ: Foraminal and extraforaminal lumbar disc herniation: diagnosis and treatment. Spine 12:577-585, 1987

10. Kang MS, Lee JW, Zhang HY, Cho YE, Park YM: Diagnosis of cervical OPLL in lateral radiograph and MRI: is it reliable? Korean J Spine 9:205-208, 2012

11. Kanno H, Endo T, Ozawa H, Koizumi Y, Morozumi N, Itoi E, et al: Axial loading during magnetic resonance imaging in patients with lumbar spinal canal stenosis: does it reproduce the positional change of the dural sac detected by upright myelography? Spine 37:E985-992, 2012

12. Kanno H, Ozawa H, Koizumi Y, Morozumi N, Aizawa T, Kusakabe $\mathrm{T}$, et al: Dynamic change of dural sac cross-sectional area in axial loaded magnetic resonance imaging correlates with the severity of clinical symptoms in patients with lumbar spinal canal stenosis. Spine 37:207-213, 2012

13. Kebaish KM, Neubauer PR, Voros GD, Khoshnevisan MA, Skolasky RL: Scoliosis in adults aged forty years and older: prevalence and relationship to age, race, and gender. Spine 36:731-736, 2011

14. Kilshaw M, Baker RP, Gardner R, Charosky S, Harding I: Abnormalities of the lumbar spine in the coronal plane on plain abdominal radiographs. Eur Spine J 20:429-433, 2011

15. Klein KM, Shiratori K, Knake S, Hamer HM, Fritsch B, TodorovaRudolph A, et al: Status epilepticus and seizures induced by iopamidol myelography. Seizure 13:196-199, 2004

16. Merkle M, Maier G, Danz S, Kaminsky J, Tatagiba MS, Hebela $\mathrm{NM}$, et al: The value of dynamic radiographic myelography in addition to magnetic resonance imaging in detection lumbar spinal canal stenosis: a prospective study. Clin Neurol Neurosurg 143: 4-8, 2016

17. Morita M, Miyauchi A, Okuda S, Oda T, Iwasaki M: Comparison between MRI and myelography in lumbar spinal canal stenosis for the decision of levels of decompression surgery. J Spinal Disord Tech 24:31-36, 2011

18. Nachemson A: Adult scoliosis and back pain. Spine 4:513-517, 1979

19. Penning L, Wilmink JT: Posture-dependent bilateral compression of L4 or L5 nerve roots in facet hypertrophy. A dynamic CT-myelographic study. Spine 12:488-500, 1987

20. Pérennou D, Marcelli C, Hérisson C, Simon L: Adult lumbar scoliosis. Epidemiologic aspects in a low-back pain population. Spine 19:123-128, 1994

21. Postacchini F, Massobrio M: Outpatient lumbar myelography. Analysis of complications after myelography comparing outpatients with inpatients. Spine 10:567-570, 1985

22. Sandow BA, Donnal JF: Myelography complications and current practice patterns. AJR Am J Roentgenol 185:768-771, 2005

23. Sasaki K, Hasegawa K, Shimoda H, Keiji I, Homma T: Can recumbent magnetic resonance imaging replace myelography or computed tomography myelography for detecting lumbar spinal stenosis? Eur J Orthop Surg Traumatol 23 Suppl 1:S77-83, 2013

24. Schwab F, Dubey A, Gamez L, El Fegoun AB, Hwang K, Pagala $\mathrm{M}$, et al: Adult scoliosis: prevalence, SF-36, and nutritional parame- 
ters in an elderly volunteer population. Spine 30:1082-1085, 2005

25. Shiban E, von Lehe M, Simon M, Clusmann H, Heinrich P, Ringel F, et al: Evaluation of degenerative disease of the lumbar spine: MR/MR myelography versus conventional myelography/post-myelography CT. Acta Neurochir (Wien) 158:1571-1578, 2016

26. Singh S, Rajpal C, Nannapeneni S, Venkatesh S: Iopamidol myelography-induced seizures. MedGenMed 7:11, 2005

27. Suess O, Stendel R, Baur S, Schilling A, Brock M: Intracranial haemorrhage following lumbar myelography: case report and review of the literature. Neuroradiology 42:211-214, 2000

28. Urrutia J, Diaz-Ledezma C, Espinosa J, Berven SH: Lumbar scolio- sis in postmenopausal women: prevalence and relationship with bone density, age, and body mass index. Spine 36:737-740, 2011

29. Willén J, Danielson B, Gaulitz A, Niklason T, Schönström N, Hansson T: Dynamic effects on the lumbar spinal canal: axially loaded CT-myelography and MRI in patients with sciatica and/or neurogenic claudication. Spine 22:2968-2976, 1997

30. Xu L, Sun X, Huang S, Zhu Z, Qiao J, Zhu F, et al: Degenerative lumbar scoliosis in Chinese Han population: prevalence and relationship to age, gender, bone mineral density, and body mass index. Eur Spine J 22:1326-1331, 2013 